\title{
Wahlmöglichkeiten und Wettbewerb in der Krankenhausversorgung aus gesundheitsökonomischer Sicht - Kommentar zum Bericht über Deutschland
}

\author{
Günter Neubauer
}

1. Das deutsche Krankenhausversorgungssystem als planwirtschaftlicher Entwurf 264

2. Transformation von der Planwirtschaft zu einer Wettbewerbswirtschaft 266

3. Ist freier Wettbewerb für die Krankenhausversorgung zielführend? 266

4. Krankenhausversorgung und Wettbewerbsrecht 268

5. Zweiteilung des Vertragsmarktes durch kollektive und selektive Verträge 269

6. Ausblick: Das Krankenhausfinanzierungsreformgesetz von 2008/9 272 
1. Das deutsche Krankenhausversorgungssystem als planwirtschaftlicher Entwurf

Betrachtet man die Geschichte des deutschen Krankenhausversorgungssystems der letzten 25 Jahre, so kann man feststellen, dass dieses grundsätzlich nach einem planwirtschaftlichen Modellansatz aufgebaut ist. Kennzeichen dieses planwirtschaftlichen Ansatzes sind, dass die Krankenhäuser in einen staatlichen Angebotsplan eingebunden sind, der ihre betriebliche Entscheidungsfreiheit deutlich einengt. So sind sowohl der Standort, die Leistungsbreite als auch die Bettenzahl vorgegeben. Ungewöhnlich ist, dass an diesem planwirtschaftlichen Versorgungssystem nicht nur öffentliche Krankenhäuser, sondern auch freigemeinnützige Krankenhäuser und auch private, gewinnorientierte Krankenhäuser teilnehmen. Einschränkend muss man jedoch hinzufügen, dass die gewinnorientierten Krankenhäuser in der Ausgangsplanung des Jahres 1972 kaum eine Rolle spielten. Im Zentrum standen die öffentlichen Krankenhäuser und, je nach Geschichte des Bundeslandes, auch die freigemeinnützigen.

Abb. 1: Die staatlich geplante Krankenhaushierarchie

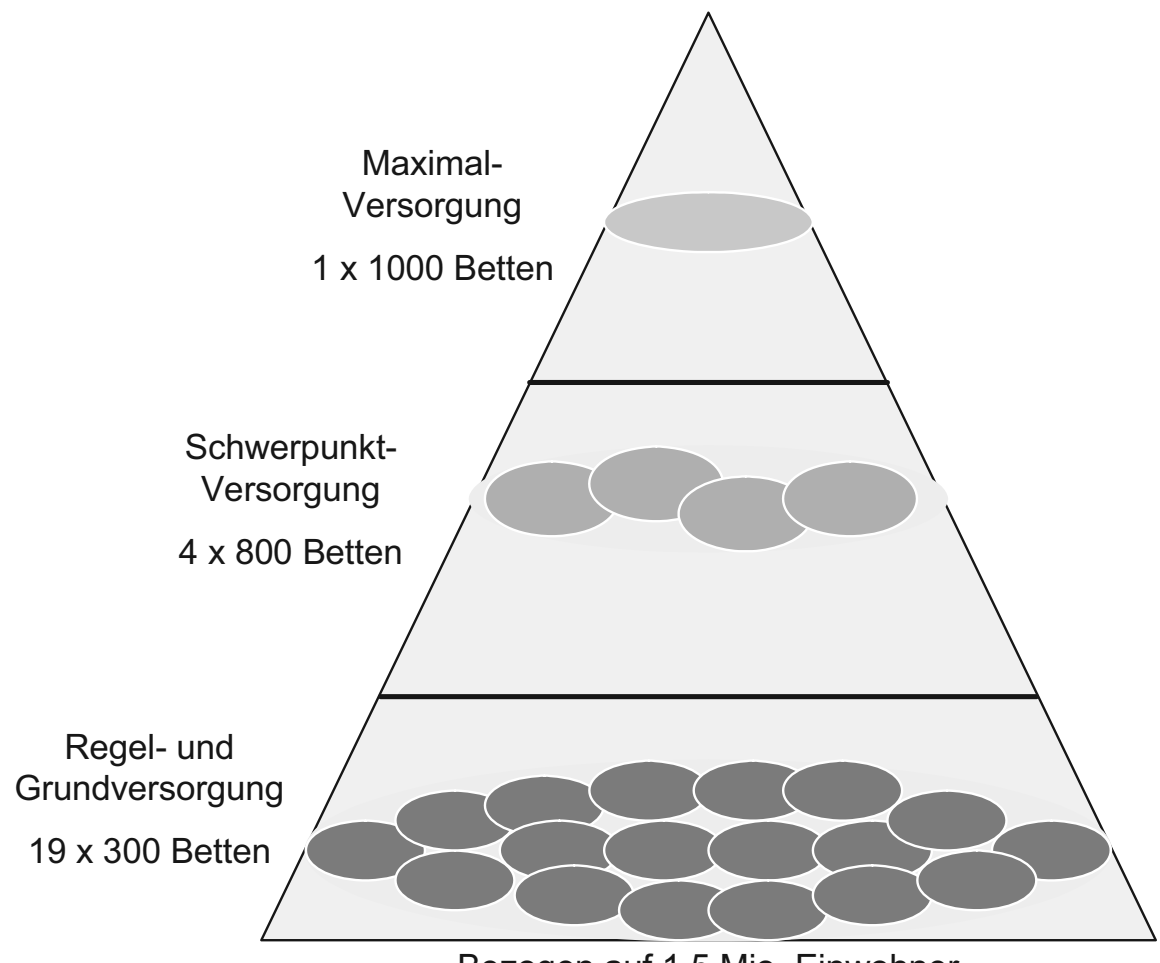

Bezogen auf 1,5 Mio. Einwohner

Ein weiteres planwirtschaftliches Kennzeichen neben der staatlichen Krankenhausplanung ist, dass das Entgeltsystem ebenfalls von strikten staatlichen Vorgaben beherrscht wird. Zum einen werden die Krankenhäuser bei ihren Investitionsplanungen 
einem staatlichen Investitionsplan unterworfen. Aus ökonomischer Sicht liegt eine staatliche Investitionslenkung vor, mittels der der Angebotsplan in konkrete Behandlungskapazitäten umgesetzt werden soll. Die laufenden Kosten, die seit 1972 von den Krankenkassen übernommen werden, sind ursprünglich, und eigentlich auch noch bis heute, auf dem so genannten Selbstkostendeckungsprinzip aufgebaut. Ökonomisch bedeutet das Selbstkostendeckungsprinzip, dass man davon ausgeht, dass Krankenhäuser ihre durchschnittlichen Kosten über die Vergütung abdecken sollen. Dies impliziert, dass Krankenhäuser im Durchschnitt keine Verluste, aber auch keine Gewinne machen. Daraus lässt sich erkennen, dass dauerhaft gewinnorientierte Krankenhäuser in diesem System die Versorgung nicht alleine übernehmen könnten.

Abb. 2: Das System der Krankenhausversorgung in Deutschland

Angebotsseite

Steuerung

Nachfrageseite

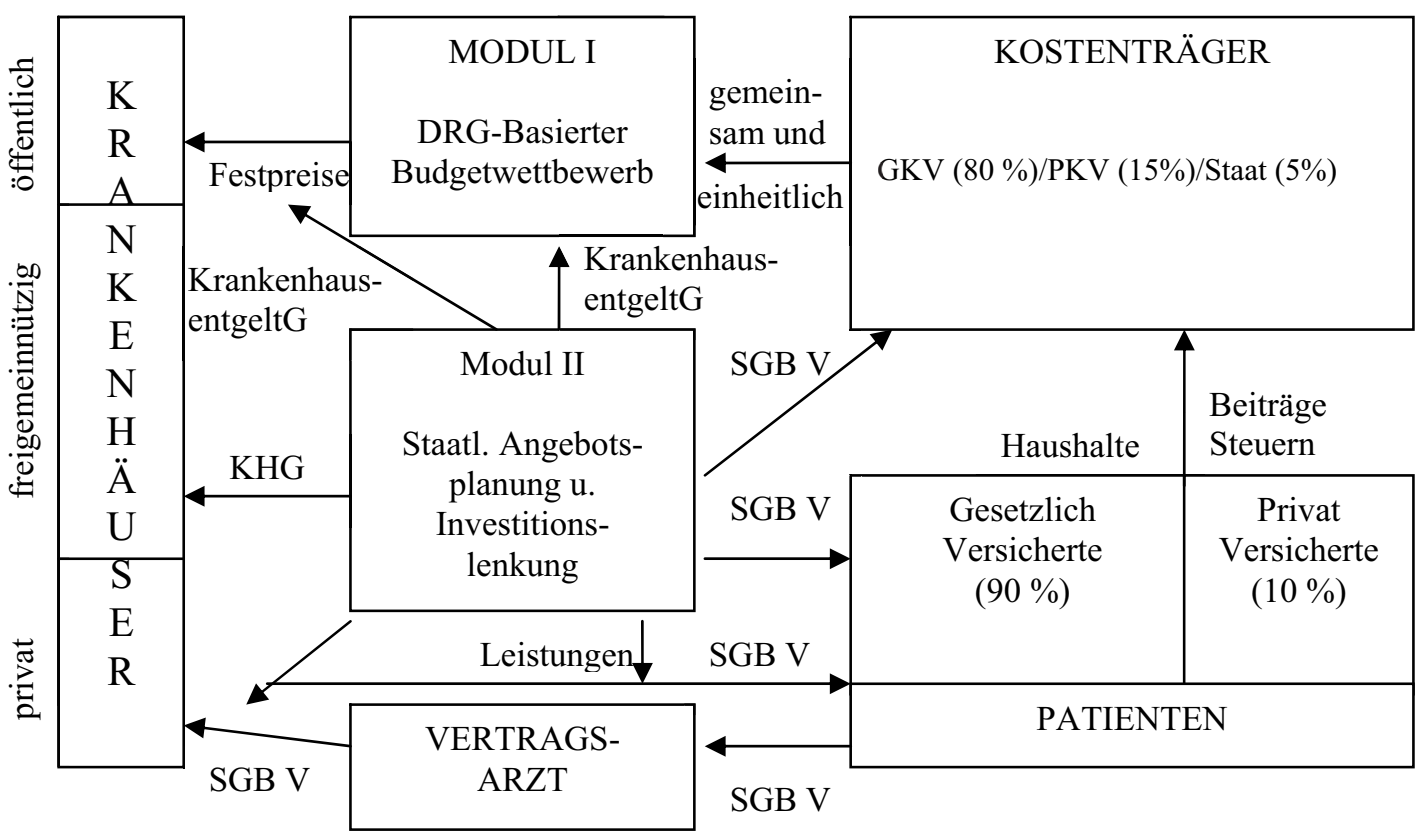

Die Krankenkassen schließlich sind bei der Auswahl ihrer Vertragspartner nicht frei, sondern an den staatlichen Krankenhaus- und Investitionsplan gebunden. Jedes Plankrankenhaus hat einen einklagbaren Anspruch auf einen Versorgungsvertrag mit den jeweiligen Krankenkassen. Es gibt somit für die Plankrankenhäuser kein echtes Marktrisiko in dem Sinne, dass sie keinen Vertragspartner finden, der kontrahiert. Andererseits haben Krankenhäuser außerhalb des Angebotsplanes, von Ausnahmen abgesehen, keinen Zutritt zur Versorgung von 90\% der Patienten. Lediglich die Privatversicherten und Selbstzahler, etwa $10 \%$ des Marktes stehen ihnen offen, wobei Letztere auch $\mathrm{Zu}$ - 
gang zu den Plankrankenhäusern haben. Alles zusammen genommen gilt, dass die deutsche Krankenhausversorgung der Idee und Ausgestaltung nach ein staatlich geplantes System ist, das die konstitutiven Merkmale einer Planwirtschaft, nämlich staatliche Gebote und Verbote statt Vertragsfreiheit, begrenzte Eigentumsrechte statt Privateigentum und eine staatliche Planbehörde statt einer Wettbewerbsaufsicht aufweist.

\section{Transformation von der Planwirtschaft zu einer Wettbewerbswirtschaft}

In dem vorliegenden Band wird mit Recht darauf hingewiesen, dass die deutsche Krankenhausversorgung sich in einem Transformationsprozess befindet. Aus rechtlicher Sicht ist dieser dadurch gekennzeichnet, dass Vorschriften des Sozialrechtes mehr und mehr mit Vorschriften aus dem Wirtschaftsrecht, insbesondere dem Wettbewerbsrecht, gemischt werden. Diese Entwicklung ist seit etwa 15 Jahren besonders deutlich zu beobachten.

Aus ökonomischer Sicht kann man feststellen, dass sich der planwirtschaftliche Ansatz mehr und mehr als suboptimal erweist, und daher der Staat und andere wichtige Akteure die Zukunft in einem stärker wettbewerblich orientierten Krankenhausversorgungssystem sehen. Allerdings wird in dem vorliegenden Band sehr deutlich herausgearbeitet, dass kein freies Wettbewerbssystem angestrebt wird, sondern letztlich immer nur ein regulierter Wettbewerb das Ziel ist.

Was sind nun die Kennzeichen eines regulierten Wettbewerbs? Auch auf diese Frage wird im vorliegenden Band eine relativ deutliche und klare Antwort gegeben. Der regulierte Wettbewerb zeichnet sich durch seinen instrumentalen Charakter aus. Der Staat setzt Wettbewerb ein, um von ihm vorgegebene Ziele effizienter erreichen zu können. Wenn das Kennzeichen des freien Wettbewerbs ist, dass das Ergebnis nicht vorhersehbar ist, so ist das Kennzeichen des regulierten Wettbewerbs, dass das Ziel vorgegeben ist und der Wettbewerb letztlich instrumentellen Charakter hat. Regulierter Wettbewerb bedeutet also, dass nicht die freien Marktkräfte zum Zuge kommen, sondern in erster Linie staatliche Regulative so gesetzt werden, dass die Ergebnisse des regulierten Wettbewerbs zu den staatlich gewollten Ergebnissen führen.

\section{Ist freier Wettbewerb für die Krankenhausversorgung zielführend?}

Die vorliegende Arbeit geht davon aus, dass freier Wettbewerb für die Krankenhausversorgung aus sozialrechtlichen und normativen Gründen nicht zulässig ist bzw. nicht zielführend sein kann. Diese Auffassung ist in Deutschland heute vorherrschend, wenngleich es mehr und mehr Konfliktpunkte an dieser Stelle gibt. 
Ein Kennzeichen dabei ist, dass den Patienten heute freies Wahlrecht bei einer Krankenhausbehandlung eingeräumt wird. Dieses freie Wahlrecht ist in einer Planwirtschaft insofern kontraproduktiv, als durch die freien Wahlmöglichkeiten der Patienten die geplanten Versorgungskapazitäten der Krankenhäuser konterkariert werden könnten. Grundsätzlich ist nämlich der Kassenpatient an die Einweisung eines Vertragsarztes gebunden. Dieser wiederum ist gehalten, den Patienten in das jeweils medizinisch adäquate Krankenhaus einzuweisen, dabei ist er auch dem Gebot der Wirtschaftlichkeit verpflichtet.

Abb. 3: Aushöhlung der Planungshierarchie durch Wettbewerb

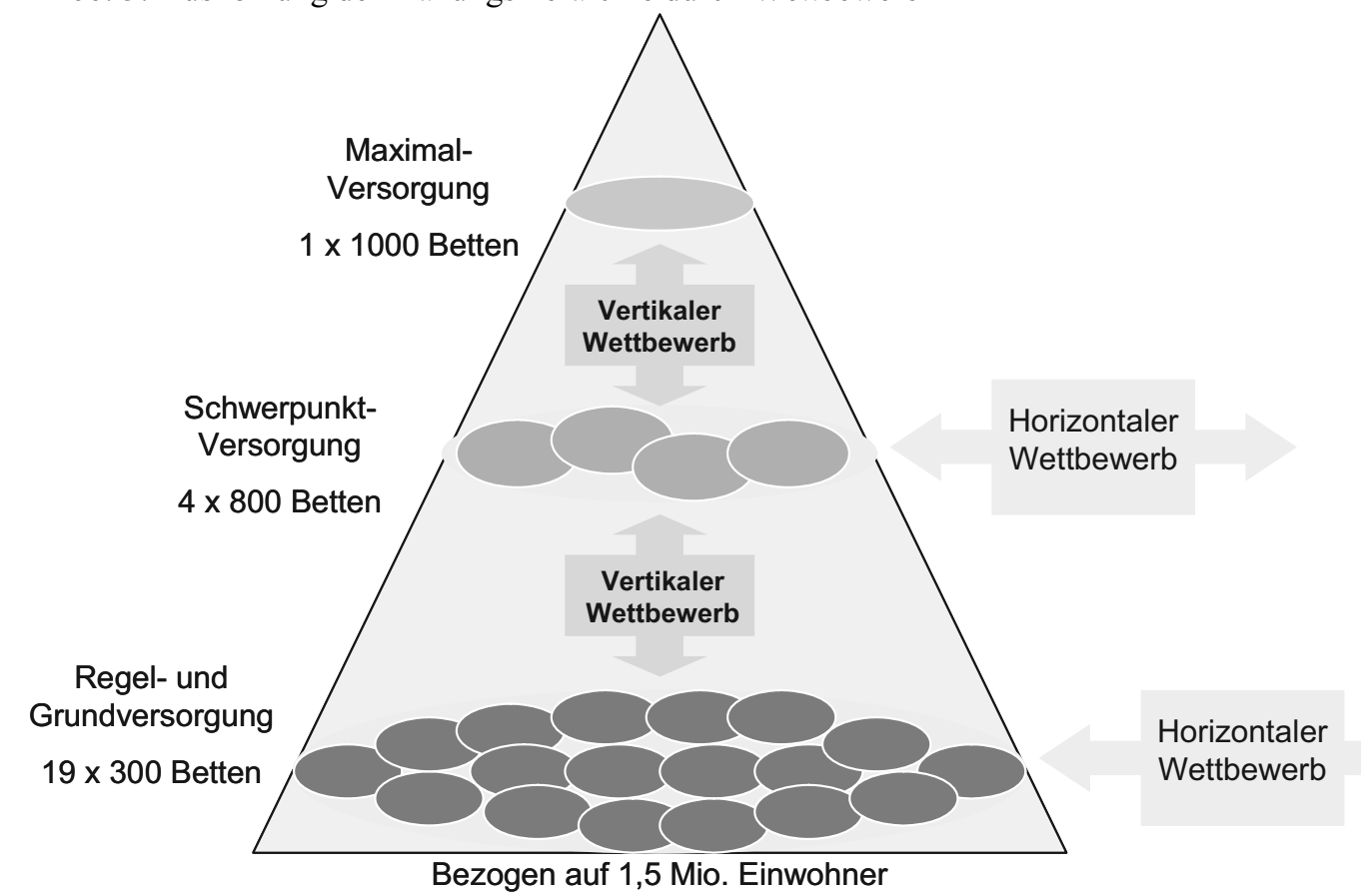

Nun ist in einem System von einheitlichen Vergütungen bzw. Preisen die Wirtschaftlichkeit nicht an der Preishöhe abzulesen. Gleichwohl unterstellt man, dass Patienten die Leistungen wie auch insbesondere die Leistungsqualität eines Krankenhauses vergleichend beurteilen können. Akzeptiert man aber diesen Schritt, dann ist der Schritt zu einem freiheitlichen Wettbewerb nicht mehr weit. Schließlich ist anzufügen, dass Patienten über die modernen Möglichkeiten der Telekommunikation auch tatsächlich mehr und mehr über die Krankenhäuser Informationen erhalten, wobei ihnen ihre Krankenkassen mit entsprechenden Angeboten zur Seite stehen.

Auch das Vordringen von selektiven Verträgen beinhaltet, dass der Versicherte die Versorgungsinhalte der selektiven Verträge bewerten und vergleichen kann. Andernfalls wäre es nicht sinnvoll, den Patienten bzw. Versicherten ein freies Wahlrecht hierzu einzuräumen. 


\section{Krankenhausversorgung und Wettbewerbsrecht}

Eine große Rolle spielt in der vorliegenden Abhandlung, aber auch in dem tatsächlichen Geschehen derzeit, die Frage, inwieweit Krankenhäuser, die sich aus ökonomischem Druck zu größeren Einheiten zusammenschließen, dem Kartellrecht unterliegen. Grundsätzlich hat im System der staatlichen Krankenhausplanung jedes Krankenhaus eine vom Krankenhausplan her vorgesehene regionale Monopolstellung. Schließlich ist die Krankenhausplanung so aufgebaut, dass die Krankenhäuser flächendeckend die Bevölkerung versorgen, aber eben nicht sich gegenseitig Konkurrenz machen. Die jeweiligen $\mathrm{zu}$ versorgenden Gebiete sind planerisch voneinander abgegrenzt. Entsprechend sind Standort, die Zahl der Betten und das medizinische Versorgungsangebot der einzelnen Krankenhäuser vorgegeben. Wenn nun Krankenhäuser sich über diese ihnen zugeordneten Versorgungsbereiche hinweg zusammenschließen, entsteht im Grunde genommen kein größeres Monopol, sondern allenfalls eine neue Versorgungsregion, in der die Versorgung neu strukturiert und in der Regel effizienter gestaltet wird.

Dass das Kartellamt diese Meinung nicht teilt, sondern davon ausgeht, dass Krankenhäuser marktbeherrschende Stellungen erreichen können, bedeutet, dass das Kartellamt letztlich den vorgegebenen Krankenhausplan als nicht bzw. nicht mehr zwingend für die einzelnen Krankenhäuser ansieht. Und tatsächlich ist es möglich, dass Krankenhäuser durch Zusammenschluss sich in eine Art Monopolfunktion überregionaler Art bringen, die jenseits der im Krankenhausplan vorgesehenen Position liegt. Da aber diese Krankenhäuser allesamt einem Versorgungsgebot zu vorgegebenen Preisen unterliegen, und umgedreht die Krankenkassen ihnen auch einen Versorgungsvertrag geben müssen, ist nicht schnell zu erkennen wo hier die Gefahr einer Monopolisierung droht. Auch hier scheint das Kartellamt längerfristig Preiswettbewerb zu erwarten, mit den dann schädlichen Folgen eines Monopolisten auf die Konkurrenten. Andernfalls wäre es nicht zu rechtfertigen, dass das Kartellrecht in dieser strikten Form angewendet wird.

Unabhängig bleibt davon die im Kartellrecht immer strittige Frage der Marktabgrenzung ungelöst. Die Frage, wie weit Patienten bereit sind zu wandern, und damit das jeweils zu betrachtende wettbewerbsrelevante Marktgebiet ausdehnen, ist offen und letztlich auch von der jeweiligen Fachdisziplin abhängig. In der Notfallversorgung ist sicherlich die regionale Zugänglichkeit von höherer Bedeutung als bei elektiven Behandlungen, bei denen heute die Bevölkerung auch weite Wegstrecken in Kauf nimmt, wenn sie denn meint ein besseres, d. h. medizinisch höher stehendes Angebot erreichen zu können.

In der Tendenz kann vorher gesagt werden, dass der anhaltende Kostendruck die Konzentration der Krankenhäuser zu größeren Unternehmenseinheiten vorantreiben wird. Die bisherige betriebliche Konzentration innerhalb der Kommunen wird hierbei deshalb nicht wahrgenommen, weil die kommunalen Eigentümer ihre bisher betrieblich eigenständigen Krankenhäuser in ein öffentliches Unternehmen mit zentraler Leitung 
zusammenfassen. Ähnliches bahnt sich auch im frei-gemeinnützigen Bereich an. Selbstverständlich werden für die Patienten aber auch für die Krankenkassen die Wahlmöglichkeiten begrenzt, wenn fünf kommunale Häuser ihre Leistungsspektren abstimmen und mit einem Vorstand die Budgetverhandlungen mit den Kassen führen. Selbst dann, wenn die rund 2000 Krankenhausbetriebe in Deutschland von rund 100 Unternehmen geführt werden, kann dennoch der Wettbewerb noch intensiver sein als heute. Der oligopolitische Wettbewerb wird ohnedies von vielen Wettbewerbstheoretikern als intensiver und innovativer eingeschätzt als der polypolistische.

\section{Zweiteilung des Vertragsmarktes durch kollektive und selektive Verträge}

Im vorgelegten Bericht spielt das selektive Kontrahieren in den verschieden praktizierten Formen eine bedeutende Rolle. Bezeichnet man die Vertragsbeziehungen zwischen den Krankenkassen und den Leistungserbringern allgemein als Vertragsmarkt, so ist seit etwa 10 Jahren, insbesondere aber mit den Reformen 2000, 2004 und 2007 eine Zweiteilung zu beobachten. Einerseits gibt es die gemeinsamen und einheitlichen (kollektiven) Verhandlungen zwischen den öffentlich-rechtlichen Verbänden der Krankenkassen und den entsprechenden Verbänden der Leistungserbringer. Andererseits werden den einzelnen Krankenkassen und privatrechtlich organisierten Leistungserbringergruppen Möglichkeiten eröffnet, Vertragspartner und Vertragsinhalte kassenindividuell zu gestalten, wobei die generelle Kontrahierungspflicht der Kassen gegenüber den Plankrankenhäusern noch bestehen bleibt.

Aus ökonomischer Sicht wird damit ein Kompetenzstransfer aus dem öffentlichrechtlichen Versorgungssektor in einen privat-rechtlichen Wettbewerbsbereich eingeleitet. Die Akteure sind damit einmal außerhalb und einmal innerhalb des Wettwerbsrechts tätig. Und an dieser Stelle gibt es auch entsprechenden juristischen Klärungsbedarf, der im Abschnitt 3.1 des Berichtes thematisiert wird.

Es ist wichtig zu verstehen, dass es zunächst nur um eine Spaltung des Vertragsmarktes geht. Der Gesetzgeber initiiert quasi einen Substitutionswettbewerb zwischen den Regelungssystemen „kollektive vs. individuelle“ Vertragssteuerung. Der „Leistungsmarkt", das sind die Beziehungen der Patienten zu den Leistungserbringern, bleibt dem Volumen nach unverändert, doch kommt es zu gewissen strukturellen Veränderungen, wie z.B. durch DMPs. Erst wenn die Krankenkassen Wahltarife für zusätzliche Leistungen ihren Versicherten anbieten, kommt es zu einer additiven Leistungsausdehnung und damit zum unvermeidlichen Konflikt mit den privaten Krankenversicherern. 
Abb. 4: Vom kollektiven Verhandeln der Basisfallwerte ...

Länder-Krankenhaus-

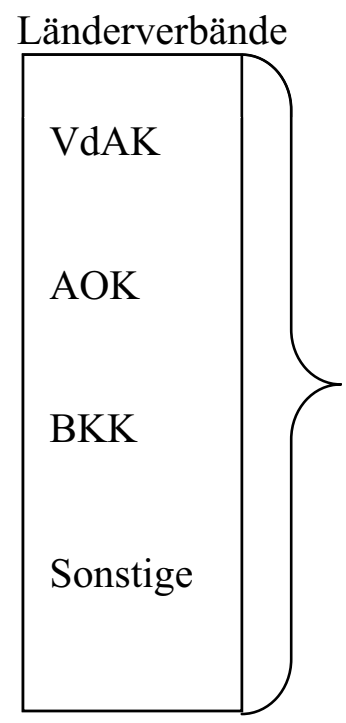

gesellschaft

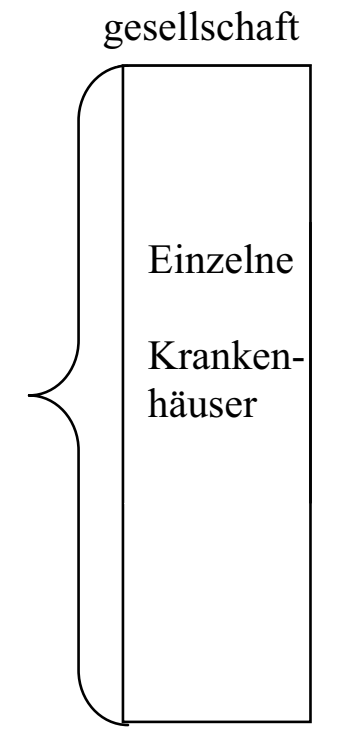

C Basisfallwert landeseinheitlich und gemeinsam

Abb. 5: ...über individuelles Verhandeln der Basisfallwerte...

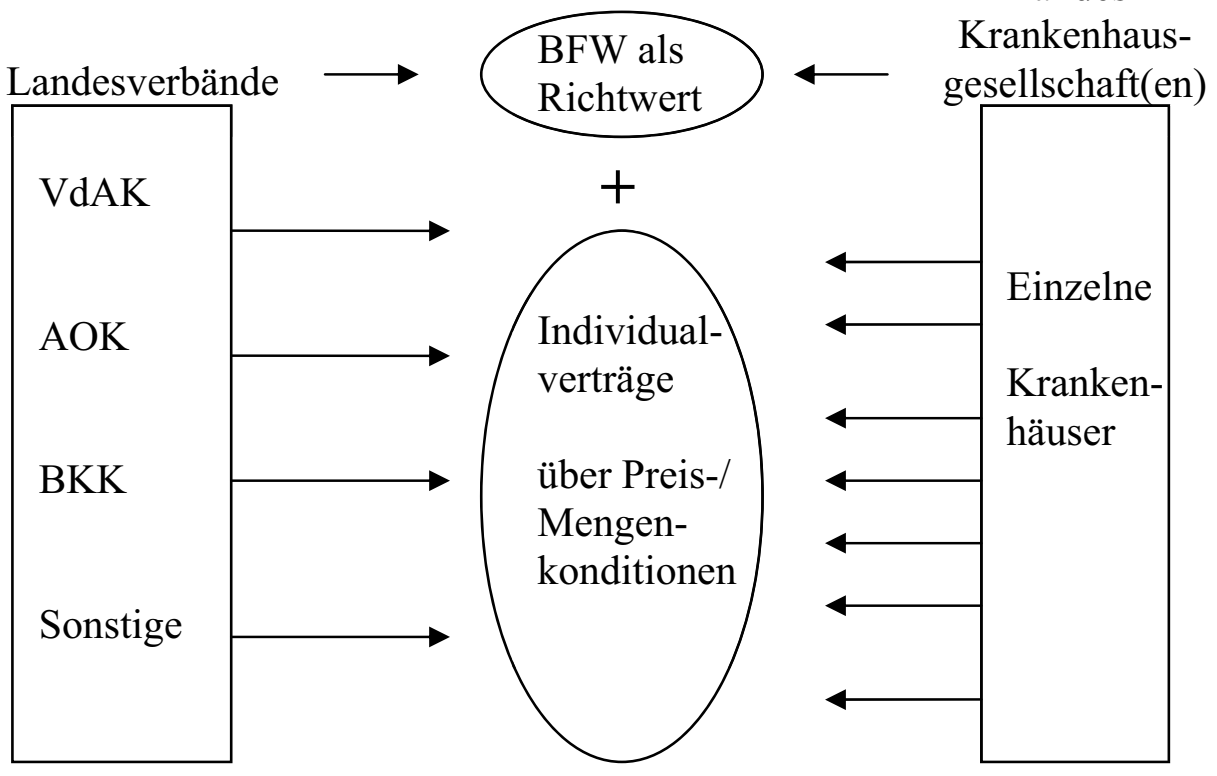


Für die Krankenhausversorgung schlagen die AOK und auch das Bundesministerium für Gesundheit auf der Referentenebene ${ }^{1}$ ein weitergehendes selektives Vertragsrecht vor: Die Notfallversorgung soll weiterhin kollektivvertraglich geregelt werden, während elektive, planbare Krankenhausleistungen auf der Definitionsebene von DRGs über Ausschreibungen der Krankenkassen selektiv an Krankenhäuser vergeben werden sollen. Damit wird die Kontrahierungspflicht der Krankenkassen teilweise aufgehoben und die Krankenhausplanung hat nur noch indikativen Charakter. Dass dieser Vorschlag kaum so ungesetzt werden kann, insbesondere weil die Planung der Länder ausgehebelt werden könnte, bleibt dahingestellt. Wichtig ist in diesem Zusammenhang, in welche Richtung die Differenzierung des Vertragsmarktes weist.

Abb. 6: ... zur selektiven Ausschreibung von elektiven Fällen?

\section{DRG: Hernien (was?)}

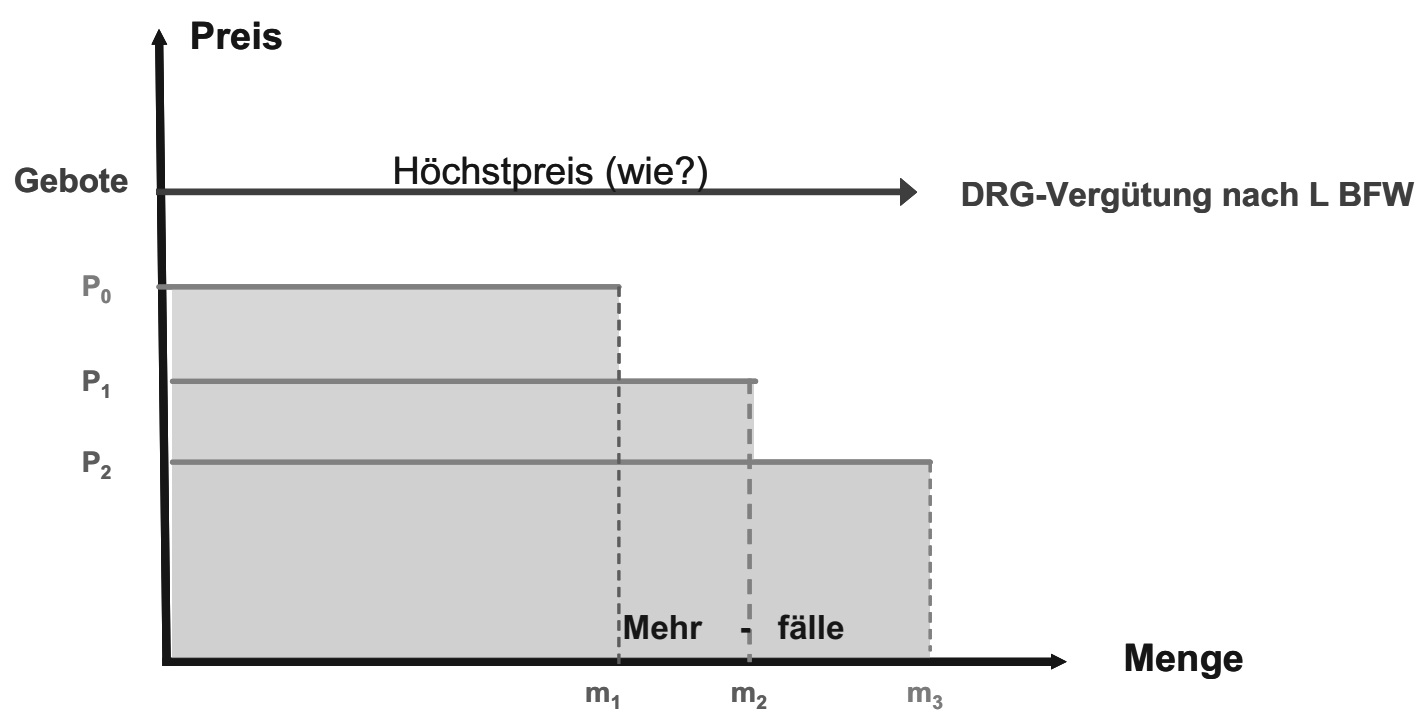

1 Vgl. G. Neubauer und A. Beivers, Krankenhaus Report 2007. 


\section{Ausblick: Das Krankenhausfinanzierungsreformgesetz von 2008/9}

Mit dem Ende der DRG-Konvergenzphase Ende des Jahres 2008 ist eine gesetzliche Wegweisung für die weitere Krankenhausfinanzierung erforderlich. Mit dem KHRG wurde eine solche Weichenstellung versucht. Doch kann schon vorab gesagt werden, dass dieses Gesetz bei weitem nicht die Hoffnungen erfüllt, die ursprünglich an es gestellt wurden. Die Große Koalition erwies sich nämlich als unfähig, zwischen den Bundesländern und dem Bundesrat eine einheitliche Meinungsbildung zustande zu bringen. Was sind nun die wesentlichen Vorgaben des KHRG?

Im Bereich der Vergütung wurden durch das KHRG vor allem die Finanzierungsnöte der Krankenhäuser gelindert. So wurde die Vergütung um etwa 3 Mrd. Euro für 2009 angehoben. Gleichzeitig wurde festgelegt, dass Lohnsteigerungen der Krankenhausbeschäftigten, die oberhalb der geschätzten Grundlohnerhöhung liegen, von den Krankenkassen zusätzlich zu finanzieren sind. Schließlich wurden noch den Krankenhäusern weitere Pflegestellen bewilligt, die zur Verstärkung der personellen Infrastruktur gedacht sind. Mittelfristig ist für den Bereich der Vergütung vorgesehen, dass die Landesbasisfallwerte ab 2010 auf eine Schwankungsbreite von plus 2,5 Prozent und minus 1,5 Prozent vom Bundesdurchschnitt zusammengeführt werden. Damit wird die Konvergenzphase der Bundesländer quasi auf Bundesebene fortgesetzt. Im Hintergrund steht die Vorstellung, dass ein bundesweiter Basisfallwert die Krankenhäuser am fairsten vergütet. Es ist klar, dass sich für eine solche Lösung jene Bundesländer eingesetzt haben, die sich als „Gewinner" bezeichnen. Dies sind vor allem Schleswig-Holstein und Mecklenburg-Vorpommern, während sich die „Verlierer-Länder“, nämlich RheinlandPfalz und auch das Saarland, gegen eine solche Konvergenz wehren bzw. das Tempo der Konvergenz hinauszögern wollen. Schließlich ist auch vorgesehen, dass ab 2011 die Krankenhausvergütung, und damit die Basisfallwerte, entsprechend eines Kostenindex jeweils angepasst werden sollen und damit das bisher geltende Verhandlungsprinzip ein Stück durch eine statistische Größe ersetzt wird.

Im Bereich der staatlichen Investitionsförderung wurde versucht, Ansätze zu einer monistischen Finanzierung durchzusetzen. Doch konnte sich hier das Bundesministerium für Gesundheit nicht gegen die jeweiligen Landesministerien durchsetzen. Immerhin ist vorgesehen, dass die Pauschalförderung und die Antragsförderung in eine Förderpauschale zusammengeführt werden, die möglichst leistungsbezogen aufgebaut sein soll. Schließlich ist vorgesehen, dass einzelne Bundesländer bei einer monistischen Finanzierung auch alleine vorgehen können. Die sonstigen Regelungen des KHRG beinhalten die Forderung nach einer Weiterentwicklung bzw. Einbeziehung der Psychiatrie in das DRG-System und einige technische Regelungen für den DRG-Dauerbetrieb.

Das KHRG lässt viele wichtige Fragen unbeantwortet. Im Vergütungsbereich bleibt die Antwort darauf, ob eine flexible Vergütung oder staatliche Festpreise das Ziel sind, im Ungewissen. Schließlich wird auch die Frage der Mengensteuerung nicht beantwor- 
tet. So überlässt es das Gesetz den Verhandlungspartnern, die sogenannte Mehrerlösregelung nun durch Verhandlungen entsprechend zu ersetzen. Von daher ist also die Frage, ob eine wettbewerbliche Preissteuerung oder eine staatliche Mengensteuerung dominant sein soll, weiterhin unbeantwortet.

Bei der Krankenhausplanung und Investitionsfinanzierung gibt es ebenfalls keine klaren Antworten. Zwar wird ausdrücklich darauf hingewiesen, dass sich die Länder stärker auf eine Rahmenplanung zurückziehen sollen, doch wird keine weitere Detailvorgabe oder Anregung hierzu gegeben. Schließlich ist auch die Investitionsfinanzierung weiterhin als duale Finanzierung konzipiert. Vor allem bleibt die Investitionslücke, die die bisherige staatliche Investitionsförderung hinterlassen hat, offen. Es bleibt ungelöst, wer die geschätzten 20 bis $30 \mathrm{Mrd}$. $€$ Investitionsbedarf der Krankenhäuser vor allem in den alten Bundesländern abdecken soll.

Das abschließende Urteil zum KHRG muss lauten: Es werden die richtigen Probleme diskutiert, jedoch keine Lösungen gefunden. 


\title{
Wahlmöglichkeiten und Wettbewerb in der niederländischen Krankenhausversorgung
}

\author{
Christina Walser
}

I. Grundlegendes

1. Das Gesundheitssystem der Niederlande - ein Überblick 277

2. Kernprobleme von Wettbewerb und Regulierung 279

2.1. Krankenhausversorgung $\quad 279$

2.2. Kosten der Krankenhausversorgung $\quad 279$

2.3. Normative Steuerung und politische Diskussion 281

2.3.1. Zusammenspiel von Staat und privaten Unternehmen 282

2.3.2. Flexibilisierung 283

3. Der Krankenhaussektor im Rahmen des Gesundheitssystems 284

3.1. Krankenhausversorgung 284

3.2. Organisationsstruktur des Krankenhaussektors 284

3.3. Gesetzlicher Rahmen 287

3.4. Strukturen und Institutionen der Krankenversicherungsträger 288

3.4.1. Struktur der Krankenversicherer nach dem Krankenkassengesetz bis 2005

3.4.2. Struktur der Krankenversicherer seit 2006

3.5 Rechtsverhältnisse zwischen Krankenversicherung, Leistungserbringer und Versichertem

4. Wettbewerb und Gesundheitsversorgung

4.1. Allgemeines Verständnis von Wettbewerb

4.2. Die Bedeutung von Wettbewerb im Gesundheitssystem

4.3. Gesetzlicher Rahmen

4.4. Institutionen der Wettbewerbskontrolle

II. Märkte - Wettbewerb und Regulierung in der Krankenhausversorgung 301

1. Der Verbraucher bei der Auswahl des Krankenhauses 301

$\begin{array}{ll}1.1 \text { Qualität } & 302\end{array}$

1.2. Preis $\quad 304$

2. Anreize für den Verbraucher bei der Wahl der Krankenversicherung 304

2.1 Das Recht zum Wechsel der Krankenversicherung 306

2.1.1 Preis als Anreiz $\quad 306$

2.1.2 Leistungen als Anreiz $\quad 307$

2.1.3 Zusatzversicherungen $\quad 311$

3. Das Verhältnis zwischen Krankenversicherung und Krankenhaus 312

3.1. Entwicklung der Kontrahierungsfreiheit zwischen Krankenhäusern und Krankenversicherern

$\begin{array}{ll}3.2 . & \text { Vertragsinhalt } \\ 3.313\end{array}$

$\begin{array}{ll}3.3 \text { Tarifgestaltung } & 313\end{array}$

4. Wettbewerb zwischen den Krankenhäusern $\quad 314$

$\begin{array}{ll}\text { 4.1. Rechtsform } & 314\end{array}$

4.2 Besonderheiten der selbständigen Behandlungszentren 315

$\begin{array}{ll}4.3 \text { Krankenhausplanung } & 316\end{array}$ 